\title{
A FRAGMENT OF DEPENDENCE LOGIC CAPTURING POLYNOMIAL TIME
}

\author{
JOHANNES EBBING $^{a}$, JUHA KONTINEN $^{b}$, JULIAN-STEFFEN MÜLLER $^{c}$, \\ AND HERIBERT VOLLMER ${ }^{d}$ \\ ${ }^{a, c, d}$ Leibniz Universität Hannover, Theoretical Computer Science, Appelstr. 4, 30167 Hannover, Ger- \\ many. \\ e-mail address: \{ebbing,mueller,vollmer\}@thi.uni-hannover.de \\ ${ }^{b}$ University of Helsinki, Department of Mathematics and Statistics, P.O. Box 68, 00014 Helsinki, \\ Finland. \\ e-mail address: juha.kontinen@helsinki.fi
}

\begin{abstract}
In this paper we study the expressive power of Horn-formulae in dependence logic and show that they can express NP-complete problems. Therefore we define an even smaller fragment $\mathrm{D}^{*}$-Horn and show that over finite successor structures it captures the complexity class $\mathrm{P}$ of all sets decidable in polynomial time. Furthermore, we show that the open $\mathrm{D}^{*}$-Horn-formulae correspond to the negative fragment of $\mathrm{SO} \exists$-Horn.
\end{abstract}

\section{INTRODUCTION}

Dependence logic, D, extends first-order logic by dependence atomic formulae

$$
\operatorname{dep}\left(t_{1}, \ldots, t_{n}\right)
$$

the meaning of which is that the value of the term $t_{n}$ is functionally determined by the values of $t_{1}, \ldots, t_{n-1}$. The semantics of $\mathrm{D}$ is defined in terms of sets of assignments (teams) instead of single assignments as in first-order logic. While in first-order logic the order of quantifiers solely determines the dependence relations between variables, in dependence logic more general dependencies between variables can be expressed. Historically dependence logic was preceded by partially ordered quantifiers (Henkin quantifiers) of Henkin [9], and Independence-Friendly (IF) logic of Hintikka and Sandu [10. It is known that both IF logic and dependence logic are equivalent to existential second-order logic $\mathrm{SO} \exists$ in expressive power. From the point of view of descriptive complexity theory, this means that dependence logic captures the class NP.

2012 ACM CCS: [Theory of computation]: Computational complexity and cryptography; Logic.

Key words and phrases: Dependence logic, Horn-formulae, computational complexity, descriptive complexity.

${ }_{a, b, c, d}$ This paper was supported by a grant from DAAD within the PPP programme, by DFG grant VO 630/6-2 and grants 264917 and 275241 of the Academy of Finland.

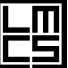

드 LOGICAL METHODS IN COMPUTER SCIENCE
DOI:10.2168/LMCS-10(3:3)2014

(C) J. Ebbing, J. Kontinen, J.-S. Müller, and H. Vollmer

(C) Creative Commons 
The framework of dependence logic has turned out be flexible to allow interesting generalizations. For example, the extensions of dependence logic in terms of so-called intuitionistic implication and linear implication was introduced in [1]. In [14 it was shown that extending $\mathrm{D}$ by the intuitionistic implication makes the logic equivalent to full second-order logic SO. On the other hand, in [2] the extension of D by a majority quantifier was defined and shown to capture the Counting Hierarchy. Furthermore, new variants of the dependence atomic formulae have also been introduced in [8, 6], and [4.

In this paper we study certain fragments of dependence logic. While it is known that D captures the class NP, the complexity of various syntactic fragments of D are not yet fully understood. Some work has been done in this direction:

- All sentences of D can be transformed to a form

$$
\forall \bar{x} \exists \bar{y}\left(\bigwedge_{i} \operatorname{dep}\left(\bar{z}_{i}, w_{i}\right) \wedge \psi\right)
$$

where $\psi$ is quantifier-free first-order formula 13 .

- In formulae of form (1.2), the use of those variables depending on others can even further be restricted; in a sense, only Boolean information in form of equality tests is needed. We will introduce this fragment $\mathrm{D}^{*}$ formally and show that it is as expressive as $\mathrm{D}$.

- The fragments of $\mathrm{D}$ defined either by restricting the number of universal quantifiers or the arity of dependence atoms in sentences were mapped to the corresponding sublogics of $\mathrm{SO} \exists$ in [3]. Making use of the well-known time hierarchy theorem this implies a strict hierarchy of fragments within D.

- The existential sentences of D collapse to FO [3], whereas the universal sentences can define NP-complete problems.

The last remark above follows from the result of [11] showing that the question of deciding whether a team $X$ satisfies $\phi$, where

$$
\phi=\operatorname{dep}(x, y) \vee \operatorname{dep}(u, v) \vee \operatorname{dep}(u, v)
$$

is NP-complete and from the observation that $\phi$ can be translated to an equivalent universal sentence of D (see the proof of Lemma 5.3).

In this paper our main objects of study are Horn fragments of D. In analogy to (1.2) we first define D-Horn to be the set of formulae of the form:

$$
\phi=\forall \bar{x} \exists \bar{y}\left(\bigwedge_{i} \operatorname{dep}\left(\bar{z}_{i}, w_{i}\right) \wedge \bigwedge_{j} C_{j}\right),
$$

where the $C_{j}$ are clauses, i.e., disjunctions of atomic and negated atomic FO-formulae, that contain at most one positive formula with an occurrence of an existentially quantified variable. While we will show that this fragment essentially is as expressive as full dependence logic (i.e., it can express NP-complete problems), we will prove that a slightly more restricted fragment, denoted by $\mathrm{D}^{*}$-Horn and obtained from D-Horn in exactly the same way as $\mathrm{D}^{*}$ is obtained from $\mathrm{D}$, corresponds over finite successor structures to the class of second-order Horn formulae which, by a famous result by Grädel [7], are known to capture $\mathrm{P}$ over finite successor structures. The result of [7] thus allows us to conclude that the sentences of $\mathrm{D}^{*}$-Horn also capture $\mathrm{P}$. An interesting question is whether $\mathrm{D}^{*}$-Horn can be somehow extended to approach the major open question of descriptive complexity, whether there is a logic for $\mathrm{P}$ properties of structures in the absence of a built-in ordering relation. We also consider the complexity of $\mathrm{D}^{*}$-Horn formulae with free variables and show that, over successor structures, the open $\mathrm{D}^{*}$-Horn-formulae correspond exactly to the negative 
fragment of $\mathrm{SO} \exists$-Horn. This result is analogous to a result of [12], who showed that the open D-formulae correspond exactly to the downward closed NP-properties.

We would like to point out that very recently, independent work of Galliani and Hella [?] obtained a characterization of $\mathrm{P}$ in terms of inclusion logic, a variant of dependence logic where instead of dependence atoms, so called inclusion atoms are used.

This article is organized as follows. In the next section, we introduce dependence logic and some of its basic properties. We also recall Grädel's characterization of $\mathrm{P}$ in terms of second-order Horn logic. In Sect. 3 we define our fragments of dependence logic, the Horn fragment and the strict Horn fragment. In Sect. 4 we present our characterization of P, and in Sect. 5, we consider the open formulae of $\mathrm{D}^{*}$-Horn.

\section{Preliminaries}

2.1. Dependence Logic. In this section we will define the semantics of dependence logic. Dependence logic (D) extends first order logic by new atomic formulae expressing dependencies between variables.

Definition 2.1 ([13]). Let $\tau$ be a vocabulary. The set of $\tau$-formulae of dependence logic $(\mathrm{D}[\tau])$ is defined by extending the set of $\tau$-formulae of first order logic $(\mathrm{FO}[\tau])$ by dependence atoms of the form

$$
\operatorname{dep}\left(t_{1}, \ldots, t_{n}\right)
$$

where $t_{1}, \ldots, t_{n}$ are terms.

In this paper, we only consider formulae in negation normal form; this means that negation occurs only in front of atomic formulae.

Definition 2.2. Let $\varphi$ be a dependence logic formula. We define the set $\operatorname{Fr}(\varphi)$ of free variables occurring in $\varphi$ as in first order logic with the additional rule

$$
\operatorname{Fr}\left(\operatorname{dep}\left(t_{1}, \ldots, t_{n}\right)\right)=\operatorname{Var}\left(t_{1}\right) \cup \cdots \cup \operatorname{Var}\left(t_{n}\right),
$$

where $\operatorname{Var}\left(t_{i}\right)$ is the set of variables occurring in $t_{i}$. A formula $\varphi$ with $\operatorname{Var}(\varphi)=\emptyset$ is called a sentence.

Now we define team semantics for dependence logic. Satisfaction for dependence logic formulae will be defined with respect to teams which are sets of assignments. Formally, teams are defined as follows.

Definition 2.3. Let $A$ be a set and $\left\{x_{1}, \ldots, x_{n}\right\}$ be a set of variables.

- Then a team $X$ over $A$ is a set of assignments $s:\left\{x_{1}, \ldots, x_{n}\right\} \rightarrow A$. We refer to $\left\{x_{1}, \ldots, x_{n}\right\}$ as the domain and to $A$ as the co-domain of $X$.

- The relation $\operatorname{rel}(X)$ over $A^{n}$ corresponding to $X$ is defined as follows

$$
\operatorname{rel}(X)=\left\{\left(s\left(x_{1}\right), \ldots, s\left(x_{n}\right)\right) \mid s \in X\right\}
$$

- Let $F: X \rightarrow A$ be a function, then we define

$$
\begin{aligned}
& X(F / x)=\{s(F(s) / x): s \in X\} \\
& X(A / x)=\{s(a / x): s \in X \text { and } a \in A\} .
\end{aligned}
$$


We are now able to define team semantics. In the following definition, $t^{\mathfrak{A}}\langle s\rangle$ for a term $t$ and an assignment $s$ denotes the value of $t$ under $s$ in structure $\mathfrak{A}$.

Definition 2.4. ([13]) Let $\mathfrak{A}$ be a model and $X$ a team of $A$. Then we define the relation $\mathfrak{A} \models_{X} \varphi$ as follows:

- If $\varphi$ is a first-order literal, then $\mathfrak{A} \models_{X} \varphi$ iff for all $s \in X$ we have $\mathfrak{A} \models_{s} \varphi$, where $\models_{s}$ refers to satisfaction in first-order logic.

- $\mathfrak{A} \models_{X} \operatorname{dep}\left(t_{1}, \ldots, t_{n}\right)$ iff for all $s, s^{\prime} \in X$ such that $t_{1}^{\mathfrak{A}}\langle s\rangle=t_{1}^{\mathfrak{A}}\left\langle s^{\prime}\right\rangle, \ldots, t_{n-1}^{\mathfrak{A}}\langle s\rangle=t_{n-1}^{\mathfrak{A}}\left\langle s^{\prime}\right\rangle$, we have $t_{n}^{\mathfrak{A}}\langle s\rangle=t_{n}^{\mathfrak{A}}\left\langle s^{\prime}\right\rangle$.

- $\mathfrak{A} \models_{X} \neg \operatorname{dep}\left(t_{1}, \ldots, t_{n}\right)$ iff $X=\emptyset$.

- $\mathfrak{A} \models_{X} \psi \wedge \varphi$ iff $\mathfrak{A} \models_{X} \psi$ and $\mathfrak{A} \models_{X} \varphi$.

- $\mathfrak{A} \models_{X} \psi \vee \varphi$ iff $X=Y \cup Z$ such that $\mathfrak{A} \models_{Y} \psi$ and $\mathfrak{A} \models_{Z} \varphi$.

- $\mathfrak{A} \models_{X} \exists x \psi$ iff $\mathfrak{A} \models_{X(F / x)} \psi$ for some $F: X \rightarrow A$.

- $\mathfrak{A} \models_{X} \forall x \psi$ iff $\mathfrak{A} \models_{X(A / x)} \psi$.

Above, we assume that the domain of $X$ contains the variables free in $\varphi$. Finally, a sentence $\varphi$ is true in a model $\mathfrak{A}$ (in symbols: $\mathfrak{A} \models \varphi$ ) if $\mathfrak{A} \models\{\emptyset\}$.

Let us then recall some basic properties of dependence logic that will be needed later. The following result shows that the truth of a D-formula depends only on the interpretations of variables occurring free in the formula. Below, for $V \subseteq \operatorname{Dom}(X), X \uparrow V$ is defined by

$$
X \uparrow V=\{s \uparrow V \mid s \in X\} .
$$

Theorem $2.5([13])$. Suppose $V \supseteq \operatorname{Fr}(\phi)$. Then $\mathfrak{A} \models_{X} \phi$ if and only if $\mathfrak{A} \models_{X \uparrow V} \phi$.

All formulae of dependence logic also satisfy the following strong monotonicity property called Downward Closure.

Theorem 2.6 ([13]). Let $\phi$ be a formula of dependence logic, $\mathfrak{A}$ a model, and $Y \subseteq X$ teams. Then $\mathfrak{A} \models_{X} \phi$ implies $\mathfrak{A} \models_{Y} \phi$.

Finally, we note that dependence logic is a conservative extension of first-order logic.

Definition 2.7. A formula $\phi$ of $\mathrm{D}$ is called a first-order formula if it does not contain dependence atomic formulae as subformulae.

First-order formulae of dependence logic satisfies the so-called flatness property:

Theorem 2.8 ([13]). Let $\phi$ be a first-order formula of dependence logic. Then for all $\mathfrak{A}$ and $X$ it holds that $\mathfrak{A} \models_{X} \phi$ if and only if for all $s \in X$ we have $\mathfrak{A} \models_{s} \phi$.

In order to study the expressive power of a logic in terms of computational complexity, we have to define the notion of "capturing" of a complexity class.

Definition 2.9. Let $\mathcal{O}$ be the class of all finite successor structures, i.e., all structures $\mathfrak{A}$ with $|\mathfrak{A}|=\{0, \ldots, n-1\}$ that contain, possibly among other constants and relations, the constants 0 and max and the relation $R=\{(x, x+1) \mid x \leq n-2\}$ for some $n \geq 1$.

\section{Definition 2.10.}

(1) Let $\mathcal{L}_{1}$ and $\mathcal{L}_{2}$ be two logics. Then $\mathcal{L}_{1} \equiv \mathcal{L}_{2}$ denotes that for every $\mathcal{L}_{1}$ sentence there is an equivalent $\mathcal{L}_{2}$ sentence, and vice-versa.

(2) Let $\mathcal{C}$ be complexity class and $\mathcal{L}$ be a logic. Then $\mathcal{C}$ is captured by $\mathcal{L}$, in symbols: $\mathcal{C} \equiv \mathcal{L}$, if for all problems $P \subseteq \mathcal{O}$ it holds that $P \in \mathcal{C}$ if and only if there exists an $\mathcal{L}$ sentence $\phi$ for which $P=\{\mathfrak{A} \in \mathcal{O} \mid \mathfrak{A} \models \phi\}$ holds. 
For dependence logic it is known that it captures NP, since $\mathrm{D} \equiv \Sigma_{1}^{1}[13]$ and $\Sigma_{1}^{1} \equiv \mathrm{NP}[5]$. Theorem 2.11. $\mathrm{D} \equiv \mathrm{NP}$.

As said, one cornerstone in the proof of the preceding theorem is a transformation of $\Sigma_{1}^{1}$-formulae into D-formulae. It can be observed that the appearing dependencies are of a very particular form.

Definition $2.12\left(\mathrm{D}^{*}\right)$. A Boolean dependence formula ( $\mathrm{D}^{*}$ formula) is a $\mathrm{D}$ formula of the form

$$
\phi=\forall \bar{x} \exists \bar{y}\left(\bigwedge_{1 \leq i \leq n} \operatorname{dep}\left(\bar{z}_{i}, y_{i}\right) \wedge \theta\right),
$$

where $\bar{y}=\left(y_{1}, \ldots, y_{n}\right)$ are pairwise distinct variables, $\bar{z}_{i} \subseteq \bar{x}$, and $\theta$ is an arbitrary firstorder formula, but the existentially quantified variables can only appear in atomic formulae of the form $y_{i}=0$ or $y_{i}=y_{j}$.

The next result shows that $\mathrm{D}^{*}$ is essentially as expressive as full dependence logic, even in the absence of a successor relation.

Theorem 2.13. (1) Over structures with built-in plus and times, $\mathrm{D}^{*} \equiv \mathrm{D}$.

(2) The logic $\mathrm{D}^{*}$ can define NP-complete problems even without the built-in successor relation.

Proof. It is known that NP can be captured by the class of second-order formulae of the form $\phi=\exists P_{1} \ldots \exists P_{n} \forall \bar{x} \theta$, where $\theta$ is a quantifier-free FO-formula, over structures with built-in predicates for plus and times [?]. As in Väänänen's proof we transform this into a D formula, see [13, Sect. 6.3]. It can be observed that the variables that appear as last variable in a dependence atom are only used in the form given in Def. 2.12 above. This proves the first claim.

The second claim follows from the fact that the fragment Strict NP, SNP [?], of sentences $\exists P_{1} \ldots \exists P_{n} \forall \bar{x} \theta$, where $\theta$ is quantifier free without order, translates into $\mathrm{D}^{*}$ under the same translation as used above, but SNP can define NP-complete problems.

2.2. Horn Logic. In this section we introduce first and second-order Horn logic and discuss their expressive power.

Definition 2.14 (FO-Horn). A clause is a disjunction of atomic and negated atomic formulae, including $\perp$ and $T$. A Horn clause is a clause with at most one non-negated atom.

Definition 2.15 (SO-Horn, [7]). A second-order Horn formula is a second-order formula $\phi$ of the form

$$
Q_{1} P_{1} \ldots Q_{k} P_{k} \forall \bar{x} \bigwedge_{j} C_{j}
$$

where for all $i, j, Q_{i} \in\{\forall, \exists\}, P_{i}$ are relation symbols, $C_{j}$ are clauses that contain at most one positive occurrence of a predicate $P_{i}$. We denote by SO-Horn the set of all second-order Horn formulae. The existential fragment of SO-Horn, denoted $\mathrm{SO} \exists$-Horn, is the fragment where all $Q_{i}=\exists$.

Theorem 2.16 ([7]). (1) SO-Horn $\equiv \mathrm{SO} \exists$-Horn.

(2) $\mathrm{SO} \exists-H o r n \equiv \mathrm{P}$. 


\section{Horn Fragments of Dependence Logic}

The Horn requirement that clauses contain at most one non-negative atom can also be transferred into the context of dependence logic.

Definition 3.1 (D-Horn). A D-Horn formula is a D formula of the form

$$
\phi=\forall \bar{x} \exists \bar{y}\left(\bigwedge_{1 \leq i \leq n} \operatorname{dep}\left(\bar{z}_{i}, y_{i}\right) \wedge \bigwedge_{j} C_{j}\right)
$$

where $\bar{y}=\left(y_{1}, \ldots, y_{n}\right)$ are pairwise distinct variables, $\bar{z}_{i} \subseteq \bar{x}$, and each clause $C_{j}$ contains at most one positive atomic formula with an occurrence of an existentially quantified variable.

As we will later show, the D-Horn fragment is essentially as expressive as full dependence logic, as it can express NP-complete problems, even without successor. But we will prove that a subfragment that informally uses only binary information about the dependent variables, exactly in the same way as in the logic $\mathrm{D}^{*}$ introduced above, has a possibly lower expressive power, since it corresponds to polynomial time computation.

Definition 3.2 ( $\mathrm{D}^{*}$-Horn). A Boolean D-Horn formula ( $\mathrm{D}^{*}$-Horn formula) is a D-Horn formula

$$
\phi=\forall \bar{x} \exists \bar{y}\left(\bigwedge_{i} \operatorname{dep}\left(\bar{z}_{i}, y_{i}\right) \wedge \bigwedge_{j} C_{j}\right)
$$

with the additional condition that the existentially quantified variables can only appear in atomic formulae of the form $y_{i}=0$ or $y_{i}=y_{j}$.

\section{EXPRESSIVE POWER OF THE HORN FRAGMENTS}

4.1. $\mathrm{D}^{*}$-Horn captures $\mathrm{P}$. In this section we investigate the expressive power of the fragments D-Horn and $\mathrm{D}^{*}$-Horn. We start by proving that the latter logic captures polynomial time.

Theorem 4.1. The logic $\mathrm{D}^{*}$-Horn captures the complexity class $\mathrm{P}$ on finite successor structures: $\mathrm{P} \equiv \mathrm{D}^{*}$-Horn.

We prove the theorem with the two following lemmas.

Lemma 4.2. SO-Horn $\subseteq \mathrm{D}^{*}$-Horn on finite successor structures.

Proof. By Theorem 2.16, it suffices to show the claim for SO $\exists$-Horn. Let

$$
\phi=\exists P_{1} \ldots \exists P_{n} \forall \bar{x} \bigwedge_{j} C_{j}
$$

be a $\mathrm{SO} \exists$-Horn sentence. First we replace quantification over relations by quantification over functions in $\phi$. This is achieved by coding each relation $P_{i}$ by its characteristic function. Furthermore, the clauses $C_{j}$ of $\phi$ are replaced by clauses $C_{j}^{\prime}$ acquired by replacing the atomic formulae $P_{i}(\bar{z})$ by $F_{i}(\bar{z})=0$. After these transformations, we get a sentence $\phi^{\prime}$ which is logically equivalent to $\phi$, where

$$
\phi^{\prime}=\exists F_{1} \ldots \exists F_{n} \forall \bar{x} \bigwedge_{j} C_{j}^{\prime} .
$$


In dependence logic, the functions $F_{i}$ will be translated by existentially quantified variables. For this reason, each occurrence of $F_{i}$ in $\phi^{\prime}$ has to be of the form $F_{i}\left(\bar{z}_{i}\right)$ for some unique tuple $\bar{z}_{i}$ of pairwise distinct variables. This can be accomplished analogously to Theorem 6.15 in [13. First, one by one, we replace each occurrence of every term $F_{i}(\bar{t})$, where $t=\left(t_{1}, \ldots, t_{k}\right)$, in $\phi^{\prime}$ by a new term $F_{i}(\bar{w})$, where $\bar{w}=\left(w_{1}, \ldots, w_{k}\right)$ is a fresh tuple of pairwise distinct variables quantified universally, and use the equivalence of $\bigwedge_{j} C_{j}^{\prime}\left(F_{i}(\bar{t})\right)$ and

$$
\forall \bar{w}\left(\bigwedge_{1 \leq p \leq k} w_{p}=t_{p} \rightarrow \bigwedge_{j} C_{j}^{\prime}\left[F_{i}(\bar{w}) / F_{i}(\bar{t})\right]\right)
$$

In this way, $\bigwedge_{j} C_{j}^{\prime}$ is transformed to an equivalent formula containing only simple terms of the form $F_{i}(\bar{w})$. It is easy to see that using distributivity laws of $\mathrm{FO}$, the quantifier-free part of formula (4.1) can be translated to a conjunction of clauses satisfying the condition of Definition 2.15, i.e., these transformations do not carry us outside of SO $\exists$-Horn.

Let us now assume that all occurences of the symbols $F_{i}$ are of the form $F_{i}(\bar{w})$ for some tuple $\bar{w}$ of pairwise distinct variables. We still need to ensure that for each $F_{i}$ the tuple $\bar{w}$ is unique. Suppose that this in not the case, i.e., assume that $\phi^{\prime}$ contains two occurrences $F_{i}(\bar{z})$ and $F_{i}\left(\bar{z}^{\prime}\right)$ of the same $F_{i}$ but with different tuples of variables. Now the idea is to replace all occurrences of $F_{i}\left(\bar{z}^{\prime}\right)$ by $G\left(\bar{z}^{\prime}\right)$, where $G$ is fresh symbol, and use the fact that $\phi^{\prime}$ is equivalent to:

$$
\exists F_{1} \ldots \exists F_{n} \exists G \forall \bar{x} \bigwedge_{j} C_{j}^{\prime}\left[G\left(\bar{z}^{\prime}\right) / F_{i}\left(\bar{z}^{\prime}\right)\right] \wedge\left(\bigwedge_{k} z_{k}=z_{k}^{\prime} \rightarrow F_{i}(\bar{z})=G\left(\bar{z}^{\prime}\right)\right)
$$

Again note that this transformation does not carry us outside of $\mathrm{SO} \exists$-Horn. Hence we may assume that in $\phi^{\prime}$ all occurrences of $F_{i}$ are of the form $F_{i}\left(\bar{z}_{i}\right)$ for some unique tuple $\bar{z}_{i}$ of pairwise distinct variables. We apply the result of [13, Theorem 6.15] which allows us to directly translate $\phi^{\prime}$ into $\mathrm{D}^{*}$-Horn which looks as follows:

$$
\forall \bar{x} \exists y_{1} \ldots \exists y_{n}\left(\bigwedge_{i=1}^{n} \operatorname{dep}\left(\bar{z}_{i}, y_{i}\right) \wedge \bigwedge_{j} C_{j}^{\prime}\left[y_{i} / F_{i}\left(\bar{z}_{i}\right)\right]\right) .
$$

Lemma 4.3. $\mathrm{D}^{*}$-Horn $\subseteq \mathrm{SO} \exists$-Horn on finite successor structures.

Proof. We prove the lemma by showing that every $\mathrm{D}^{*}$-Horn sentence can be translated to an equivalent $\mathrm{SO} \exists$-Horn sentence. Let

$$
\phi=\forall \bar{x} \exists y_{1} \ldots \exists y_{k}\left(\bigwedge_{i=1}^{h} \operatorname{dep}\left(\bar{z}_{i}, y_{i}\right) \wedge \bigwedge_{j} C_{j}\right)
$$

be a $\mathrm{D}^{*}$-Horn sentence. Without loss of generality, we assume that atoms $y_{j}=y_{j}$ do not appear in $\phi$. By the results of [13], this sentence is equivalent to the $\mathrm{SO} \exists$ sentence $\phi^{*}$

$$
\exists f_{1} \ldots \exists f_{k} \forall \bar{x} \bigwedge_{j} C_{j}^{*}
$$

where $C_{j}^{*}$ is defined by replacing the occurrences of $y_{i}$ by the term $f_{i}\left(\bar{z}_{i}\right)$. It now suffices to transform this sentence to an equivalent $\mathrm{SO} \exists$-Horn sentence. This is achieved as follows. For each pair $\{r, s\}$ such that either $f_{r}\left(\bar{z}_{r}\right)=f_{s}\left(\bar{z}_{s}\right)$ or $f_{s}\left(\bar{z}_{s}\right)=f_{r}\left(\bar{z}_{r}\right)$ occurs in $\phi^{*}$ we introduce a new relation symbol $P_{\{r, s\}}$. Furthermore, for each $\{r, 0\}$ such that $f_{r}\left(\bar{z}_{r}\right)=0$ 
occurs in $\phi^{*}$ a relation symbol $P_{\{r, 0\}}$ is introduced. Finally for each pair of relation symbols $P_{\{r, s\}}, P_{\{s, t\}}$ we add the new relation symbol $P_{\{r, t\}}$. All these new relation symbols have arity $|\bar{x}|$. In the following $s, r$ and $t$ range over $\{0,1, \ldots, k\}$ and atoms of the form $f_{r}\left(\bar{z}_{r}\right)=0$ are also written as $f_{r}\left(\bar{z}_{s}\right)=f_{0}\left(\bar{z}_{0}\right)$.

Now for each $C_{j}^{*}$ in $\phi^{*}$, the formula $C_{j}^{\prime}$ is defined by replacing the atoms $f_{r}\left(\bar{z}_{r}\right)=f_{s}\left(\bar{z}_{s}\right)$ and $f_{r}\left(\bar{z}_{r}\right)=0$ by the atoms $P_{\{r, s\}}(\bar{x})$ and $P_{\{r, 0\}}(\bar{x})$, respectively. Since $\phi \in \mathrm{D}^{*}$-Horn it follows immediately that

$$
\phi^{\prime}:=\exists \bar{P} \forall \bar{x} \bigwedge_{j} C_{j}^{\prime}
$$

is a $\mathrm{SO} \exists$-Horn sentence. In order to guarantee the equivalence of $\phi^{*}$ and $\phi^{\prime}$ (and hence the equivalence $\phi^{\prime}$ and $\phi$ ) we add to $\bigwedge_{j} C_{j}^{\prime}$ the following clauses:

$$
\bigwedge_{r, s, t}\left[\left(P_{\{r, s\}}(\bar{x}) \wedge P_{\{s, t\}}(\bar{x})\right) \rightarrow P_{\{r, t\}}(\bar{x})\right],
$$

axiomatizing the transitivity of identity. We also replace the quantifier prefix $\forall \bar{x}$ by $\forall \bar{x} \forall \bar{x}^{\prime}$ and add the following clauses to the formula:

$$
\bigwedge_{r} \bigwedge_{s}\left(\left(\bar{z}_{r}=\bar{z}_{r}^{\prime} \rightarrow\left(P_{\{r, s\}}(\bar{x}) \leftrightarrow P_{\{r, s\}}\left(\bar{x}^{\prime}\right)\right)\right),\right.
$$

which ensures that the identities satisfied by the term $f_{r}\left(\bar{z}_{r}\right)$ are determined by the values of the variables $\bar{z}_{r}$. It is straightforward to check that $\phi^{\prime}$, with the modifications in (4.4) and (4.5), is equivalent to a formula in $\mathrm{SO} \exists$-Horn. We will next show that, for structures $\mathfrak{A}$ with $|A| \geq k+1$, where $k$ is the number of functions $f_{i}$ in $\phi^{*}$, the sentences $\phi^{\prime}$ and $\phi^{*}$ are equivalent. Note that, by modifying the behaviour of $\phi^{\prime}$ in the finitely many structures of cardinality at most $k$, we can find a sentence of $\mathrm{SO} \exists$-Horn that is logically equivalent to $\phi^{*}$, and hence $\phi$.

The proof of the implication from $\mathfrak{A} \models \phi^{*}$ to $\mathfrak{A} \models \phi^{\prime}$ is straightforward, hence we consider only the converse implication. Suppose then that $\mathfrak{A}$ is a structure with $|A| \geq k+1$, and $\mathfrak{A} \models \phi^{\prime}$. We need to show that $\mathfrak{A} \models \phi^{*}$. It suffices to find interpretations $g_{i}: A^{\left|\bar{z}_{i}\right|} \rightarrow A$ for the function symbols $f_{i}$, for $1 \leq i \leq k$, such that

$$
\left(\mathfrak{A}, g_{1}, \ldots, g_{k}\right) \models \forall \bar{x} \bigwedge_{j} C_{j}^{*} .
$$

Since $\mathfrak{A} \models \phi^{\prime}$, there are relations $S_{\{s, r\}} \subseteq A^{|\bar{x}|}$ such that

$$
\left(\mathfrak{A},\left(S_{\{s, r\}}\right)_{s, r}\right) \models \forall \bar{x} \forall \bar{x}^{\prime} \bigwedge_{j} C_{j}^{\prime} .
$$

We define the functions $g_{i}$ as follows: $g_{i}(\bar{a})=l$, if $l$ is the smallest integer in $\{0, \ldots, i-1\}$ such that $\bar{a}$ can be extended to a tuple $\bar{a}^{\prime} \in A^{|\bar{x}|}$ such that $\bar{a}^{\prime} \in S_{\{i, l\}}$. Otherwise, $g_{i}(\bar{a})=i$. The formula (4.5) ensures that $g_{i}$ is well-defined. Now, using the fact that the relations $\left(S_{\{s, r\}}\right)_{s, r}$ satisfy the formula (4.4), it is straightforward to show that for all $\bar{a} \in A^{|\bar{x}|}$ :

$$
\left(\mathfrak{A}, g_{1}, \ldots, g_{k}\right) \models_{v} f_{s}\left(\bar{z}_{s}\right)=f_{r}\left(\bar{z}_{r}\right) \Leftrightarrow\left(\mathfrak{A},\left(S_{\{s, r\}}\right)_{s, r}\right) \models_{v} P_{\{s, r\}}(\bar{x}),
$$

where $v$ is the assignment such that $v(\bar{x})=\bar{a}$. Using (4.6), $\mathfrak{A} \models \phi^{*}$ easily follows. 
Corollary 4.4. $\mathrm{D}^{*}$-Horn restricted to formulae with $k$ universal quantifiers captures a subclass of $\operatorname{TIME}\left(n^{2 k}\right)$.

Proof. Starting with a formula $\psi \in \mathrm{D}^{*}$-Horn with $k$ universal quantifiers, we obtain a formula $\phi \in \mathrm{SO} \exists$-Horn with $\ell=2 k$ universal quantifiers as in the proof of the preceding lemma. As shown by [7, Corollary 4.2], a formula $\phi$ from SO $\exists$-Horn can be transformed into an equivalent propositional Horn formula $\phi^{\prime}$, such that the obtained formula has size $n^{\ell}$, where $n$ denotes the size of the model and $\ell$ the number of universal quantifiers in $\phi$. The claim now follows since evaluation of propositional Horn formulae is in linear time.

It is worth noting that Lemma 4.3 does not hold without the built-in successor relation, e.g., the following $\mathrm{D}^{*}$-Horn-sentence

$$
\exists y_{1} \ldots \exists y_{n} \bigwedge_{1 \leq i<j \leq n} \neg y_{i}=y_{j}
$$

is not logically equivalent to any $\mathrm{SO} \exists$-Horn-sentence since the properties definable in $\mathrm{SO} \exists$ Horn without successor are closed under taking substructures [7].

4.2. D-Horn expresses NP-complete problems. In the rest of this section we turn to the logic D-Horn. We show that it is essentially as expressive as $\mathrm{D}$ by proving that the following NP-complete problem Dominating-Set can be expressed in D-Horn.

$$
\text { Dominating-Set }=\left\{\begin{array}{l|l}
\langle(V, E), k\rangle & \begin{array}{l}
\text { there is a set } V^{\prime} \subseteq V, \mid V^{\prime} \leq k, \text { such that } \\
\text { for every } v \in V \backslash V^{\prime} \text { there is a } u \in V^{\prime} \text { with } \\
(u, v) \in E
\end{array}
\end{array}\right\} .
$$

Theorem 4.5. Dominating-Set can be defined in D-Horn, even without successor.

Proof. Consider the following formula

$$
\begin{aligned}
\phi=\forall x_{0} \forall x_{1} \forall x_{2} \exists y_{0} \exists y_{1} \exists y_{2} & \left(\operatorname{dep}\left(x_{0}, y_{0}\right)\right. \\
& \wedge \operatorname{dep}\left(x_{1}, y_{1}\right) \wedge \operatorname{dep}\left(x_{2}, y_{2}\right) \\
& \wedge\left(x_{1}=x_{2} \rightarrow y_{1}=y_{2}\right) \wedge\left(y_{1}=y_{2} \rightarrow x_{1}=x_{2}\right) \\
& \wedge E\left(x_{0}, y_{0}\right) \wedge\left(y_{0}=x_{1} \rightarrow P\left(y_{1}\right)\right) .
\end{aligned}
$$

We claim that, for $G=(V, E),\langle G, k\rangle \in$ Dominating-Set $\Longleftrightarrow\left\langle G^{*}, k\right\rangle \in$ Dominating-Set $\Longleftrightarrow\left(G^{*}, P\right) \models \phi$, where $P$ is an arbitrary unary relation that contains $k$ nodes and $G^{*}$ extends $G$ by self loops.

To see this, note that the atoms in the second and third line in (4.7) define a bijection $f$ under which $x_{1}$ is mapped to $y_{1}$ (this is analogous to the example on p. 51 in [13]). The rest of the formula ensures that every node $x_{0}$ is connected by an edge to some node $y_{0}$ and $y_{0}$ is in bijection with some element in $P$. Since there are only $k$ such elements, we express existence of a dominating set of cardinality $k$. 


\section{The Case of open formulae}

In this section we show that over successor structures the open $\mathrm{D}^{*}$-Horn-formulae correspond exactly to the negative fragment of $\mathrm{SO} \exists$-Horn.

Theorem 2.11 shows that sentences of $\mathrm{D}$ correspond to sentences of $\mathrm{SO} \exists$. Note that this result does not tell us anything about formulae of dependence logic with free variables. An upper bound for the complexity of formulae of $\mathrm{D}$ is provided by the following result.

Theorem 5.1 ([13]). Let $\tau$ be a vocabulary and $\varphi$ a $\mathrm{D}[\tau]$-formula with free variables $x_{1}, \ldots, x_{k}$. Then there is a $\tau \cup\{R\}$-sentence $\psi$ of $\mathrm{SO} \exists$, in which $R$ appears only negatively, such that for all models $\mathfrak{A}$ and teams $X$ with domain $\left\{x_{1}, \ldots, x_{k}\right\}$ :

$$
\mathfrak{A} \models_{X} \phi \Longleftrightarrow(\mathfrak{A}, \operatorname{rel}(X)) \models \psi \text {. }
$$

In [12] it was shown that also the converse holds.

Theorem 5.2 ([12]). Let $\tau$ be a vocabulary and $R$ a $k$-ary relation symbol such that $R \notin \tau$. Then for every $\tau \cup\{R\}$-sentence $\psi$ of $\mathrm{SO} \exists$, in which $R$ appears only negatively, there is a $\tau$-formula $\phi$ of $\mathrm{D}$ with free variables $x_{1}, \ldots, x_{k}$ such that, for all $\mathfrak{A}$ and $X \neq \emptyset$ with domain $\left\{x_{1}, \ldots, x_{k}\right\}$ :

$$
\mathfrak{A} \models_{X} \phi \Longleftrightarrow(\mathfrak{A}, \operatorname{rel}(X)) \models \psi .
$$

Theorem 5.2 shows that formulae of dependence logic correspond in a precise way to the negative fragment of $\mathrm{SO} \exists$ and are therefore very expressive. We will next proceed to generalize Theorems 5.1 and 5.2 to open $\mathrm{D}^{*}$-Horn formulae and the negative fragment of $\mathrm{SO} \exists$-Horn. We consider first the analogue of Theorem 5.1.

Lemma 5.3. Let $\tau$ be a vocabulary and $\varphi \in \mathrm{D}^{*}-\operatorname{Horn}[\tau]$ with free variables $z_{1}, \ldots, z_{k}$. Then there is a $\tau \cup\{R\}$-sentence $\psi$ of $\mathrm{D}^{*}$-Horn in which $R$ appears only negatively, such that for all models $\mathfrak{A}$ and teams $X$ with domain $\left\{z_{1}, \ldots, z_{k}\right\}$ :

$$
\mathfrak{A} \models_{X} \phi \Longleftrightarrow(\mathfrak{A}, \operatorname{rel}(X)) \models \psi \text {. }
$$

Proof. Suppose that $\varphi$ is of the form

$$
\forall \bar{x} \exists \bar{y}\left(\bigwedge_{i} \operatorname{dep}\left(\bar{w}_{i}, y_{i}\right) \wedge \bigwedge_{j} C_{j}\right) .
$$

As generally showed in Proposition 5.4 in $\left[3, \varphi\right.$ can be translated to a D sentence $\psi^{\prime}$ satisfying the equivalence (5.2):

$$
\psi^{\prime}:=\forall \bar{z}(\neg R(\bar{z}) \vee \varphi) .
$$

Therefore it suffices to show that $\psi^{\prime}$ is equivalent to some $\mathrm{D}^{*}$-Horn sentence $\psi$. We may assume that the variables in $\bar{x}$ and $\bar{y}$ do not appear in $\neg R(\bar{z})$, hence $\psi^{\prime}$ is equivalent to

$$
\forall \overline{z x} \exists \bar{y}\left(\left(\bigwedge_{i} \operatorname{dep}\left(\bar{w}_{i}, y_{i}\right) \wedge \bigwedge_{j} C_{j}\right) \vee \neg R(\bar{z})\right) .
$$

The proof of Lemma 3.2 in [3] shows that the following subformula of (5.3)

$$
\exists \bar{y}\left(\left(\bigwedge_{i} \operatorname{dep}\left(\bar{w}_{i}, y_{i}\right) \wedge \bigwedge_{j} C_{j}\right) \vee \neg R(\bar{z})\right)
$$

is equivalent to

$$
\exists \bar{y}\left(\bigwedge_{i} \operatorname{dep}\left(\bar{w}_{i}, y_{i}\right) \wedge\left(\bigwedge_{j} C_{j} \vee \neg R(\bar{z})\right)\right) .
$$


Therefore, the sentence $\psi^{\prime}$ is logically equivalent to the sentence

$$
\forall \overline{z x} \exists \bar{y}\left(\bigwedge_{i} \operatorname{dep}\left(\bar{w}_{i}, y_{i}\right) \wedge\left(\bigwedge_{j} C_{j} \vee \neg R(\bar{z})\right)\right) .
$$

Note that since $\left(\bigwedge_{j} C_{j} \vee \neg R(\bar{z})\right)$ is first-order, Theorem 2.8 implies that (5.4) is equivalent to the $\mathrm{D}^{*}$-Horn sentence $\psi$

$$
\psi=\forall \overline{z x} \exists \bar{y}\left(\bigwedge_{i} \operatorname{dep}\left(\bar{w}_{i}, y_{i}\right) \wedge \bigwedge_{j} C_{j}^{\prime}\right)
$$

where $C_{j}^{\prime}$ is $C_{j} \vee \neg R(\bar{z})$.

By combining Lemma 5.3 and our translation from $\mathrm{D}^{*}$-Horn to $\mathrm{SO} \exists$-Horn the following analogue of Theorem 5.1 follows.

Theorem 5.4. Let $\tau$ be a vocabulary such that $\{0, \max , R\} \subseteq \tau$, and $\varphi$ a $\mathrm{D}^{*}$-Horn $[\tau]$ formula with free variables $z_{1}, \ldots, z_{k}$. Then there is a $\tau \cup\{R\}$-sentence $\psi$ of $\mathrm{SO} \exists$-Horn, in which $R$ appears only negatively, such that for all sufficiently large models $\mathfrak{A}$ and teams $X \neq \emptyset$ with domain $\left\{z_{1}, \ldots, z_{k}\right\}$ :

$$
\mathfrak{A} \models_{X} \phi \Longleftrightarrow(\mathfrak{A}, \operatorname{rel}(X)) \models \psi .
$$

Proof. Note that if $R$ appears only negatively in $\phi$, then it will also only appear negatively in $\phi^{\prime}$, as defined in Lemma 4.3, and these sentences are equivalent for large enough structures.

Next we show that the analogue of Theorem 5.2 also holds.

Theorem 5.5. Let $\tau$ be a vocabulary such that $\{0, \max , R\} \subseteq \tau$, and $R$ a $k$-ary relation symbol such that $R \notin \tau$. Then for every $\tau \cup\{R\}$-sentence $\psi$ of SO $\exists$-Horn, in which $R$ appears only negatively, there is a $\tau$-formula $\phi$ of $\mathrm{D}^{*}$-Horn with free variables $z_{1}, \ldots, z_{k}$ such that, for all $\mathfrak{A}$ and $X \neq \emptyset$ with domain $\left\{z_{1}, \ldots, z_{k}\right\}$ :

$$
\mathfrak{A} \models_{X} \phi \Longleftrightarrow(\mathfrak{A}, \operatorname{rel}(X)) \models \psi \text {. }
$$

Proof. Suppose $\psi \in \mathrm{SO} \exists-H o r n[\tau \cup\{R\}]$ is of the form

$$
\exists P_{1} \ldots \exists P_{k} \forall \bar{y} \bigwedge_{i} C_{i} .
$$

It is easy to check that $\psi$ is logically equivalent to $\psi^{\prime}$

$$
\psi^{\prime}:=\exists R^{\prime}\left(\forall \bar{x}\left(\neg R(\bar{x}) \vee R^{\prime}(\bar{x})\right) \wedge \psi\left[R^{\prime} / R\right]\right),
$$

where in $\psi\left[R^{\prime} / R\right]$ all occurrences of $R$ are replaced by $R^{\prime}$. Now $\psi^{\prime}$ can be transformed into

$$
\varphi^{*}=\exists R^{\prime} \exists P_{1} \ldots \exists P_{k} \forall \bar{x} \forall \bar{y}\left(\bigwedge_{i} C_{i}\left[R^{\prime} / R\right] \wedge\left(\neg R(\bar{x}) \vee R^{\prime}(\bar{x})\right)\right) .
$$

By the assumption that $R$ has only negative occurrences in $\psi$ it follows that the sentence (5.6) is also in $\mathrm{SO} \exists$-Horn. Now we define the formula $\phi\left(z_{1}, \ldots, z_{k}\right)$ by first translating the sentence (5.6) into $\mathrm{D}^{*}$-Horn as in Lemma 4.2, and then replacing the subformula $\neg R(\bar{x})$ by $\vee_{1 \leq i \leq k} \neg z_{i}=x_{i}$. We note first that $\phi \in \mathrm{D}^{*}$-Horn. Furthermore, since the way $\phi$ is obtained from sentence (5.6) is the same as the translation given in [12], the formula $\phi\left(z_{1}, \ldots, z_{k}\right)$ is as wanted. 
We say that a class $D$ of structures is downwards closed with respect to $R$ if for every structure $\mathfrak{A}$ the following holds: if $\mathfrak{A} \in D$ and $Q \subseteq R^{\mathfrak{A}}$ then $\mathfrak{A}^{\prime} \in D$, where $\mathfrak{A}^{\prime}$ arises from $\mathfrak{A}$ by replacing $R^{\mathfrak{A}}$ by $Q$. As far as we know, it is an open question whether the $R$ negative fragment of $\mathrm{SO} \exists$-Horn can define all P-properties of successor structures which are downwards closed with respect to $R$. Note that for $\mathrm{SO} \exists$ and NP the analogous result holds, and hence the open formulas of dependence logic correspond exactly to the downwards closed NP properties.

\section{Conclusion}

Inspired by Grädel's second-order characterization of $\mathrm{P}$, we have studied two fragments of dependence logic D. While the first restriction to Horn formulae still yields the full power of NP, the more restricted type of Horn formulae captures the class P. Furthermore we showed that the open $\mathrm{D}^{*}$-Horn-formulae correspond exactly to the negative fragment of $\mathrm{SO} \exists$-Horn.

We conclude with the following open questions:

(1) We showed that the model checking problem for the fragment of $\mathrm{D}^{*}$-Horn with $k$ universal quantifiers can be solved in $\operatorname{TIME}\left(n^{2 k}\right)$ (see Corollary 4.4). It would be interesting to prove a converse inclusion, i.e., that $\operatorname{TIME}\left(n^{k}\right)$ can be captured by $\mathrm{D}^{*}$-Horn with $k^{\prime}$ universal quantifiers, for some constant $k^{\prime}>k$.

(2) It would be interesting to know whether the $R$-negative fragment of $\mathrm{SO} \exists$-Horn (and the open $\mathrm{D}^{*}$-Horn formulae) capture exactly all $\mathrm{P}$ properties of successor structures which are downward closed with respect to $R$.

\section{REFERENCES}

[1] Samson Abramsky and Jouko Väänänen. From IF to BI. Synthese, 167(2):207-230, 2009.

[2] Arnaud Durand, Johannes Ebbing, Juha Kontinen, and Heribert Vollmer. Dependence logic with a majority quantifier. In Supratik Chakraborty and Amit Kumar, editors, FSTTCS, volume 13 of LIPIcs, pages 252-263. Schloss Dagstuhl - Leibniz-Zentrum fuer Informatik, 2011.

[3] Arnaud Durand and Juha Kontinen. Hierarchies in dependence logic. ACM Transactions on Computational Logic (TOCL), 13(4):31, 2012.

[4] Fredrik Engström. Generalized quantifiers in dependence logic. Journal of Logic, Language and Information, 21:299-324, 2012.

[5] Ronald Fagin. Generalized first-order spectra and polynomial time recognizable sets. In R. Karp, editor, Complexity of Computations, pages 43-73. American Mathematical Society, Providence, RI, 1974.

[6] Pietro Galliani. Inclusion and exclusion dependencies in team semantics - on some logics of imperfect information. Ann. Pure Appl. Logic, 163(1):68-84, 2012.

[7] Erich Grädel. Capturing complexity classes by fragments of second-order logic. Theor. Comput. Sci., 101:35-57, July 1992.

[8] Erich Grdel and Jouko Vnnen. Dependence and independence. Studia Logica, 101(2):399-410, 2013.

[9] Leon Henkin. Some remarks on infinitely long formulas. In Infinitistic Methods, Proceedings Symposium Foundations of Mathematics, pages 167-183, Warsaw, 1961. Pergamon.

[10] Jaakko Hintikka and Gabriel Sandu. Informational independence as a semantical phenomenon. In Jens Erik Fenstad, Ivan Timofeevich Frolov, and Risto Hilpinen, editors, Logic, Methodology and Philosophy of Science VIII, volume 126, pages 571-589. Elsevier, Amsterdam, 1989.

[11] Jarmo Kontinen. Coherence and computational complexity of quantifier-free dependence logic formulas. Studia Logica, 101(2):267-291, 2013.

[12] Juha Kontinen and Jouko Väänänen. On definability in dependence logic. J. of Logic, Lang. and Inf., 18(3):317-332, 2009. 
[13] Jouko Väänänen. Dependence logic: A new approach to independence friendly logic. Number 70 in London Mathematical Society student texts. Cambridge University Press, 2007.

[14] Fan Yang. Expressing second-order sentences in intuitionistic dependence logic. Studia Logica, 101(2):323-342, 2013.

This work is licensed under the Creative Commons Attribution-NoDerivs License. To view a copy of this license, visit http://creativecommons.org/licenses/by-nd/2.0/ or send a a copy of this license, visit http://creativecommons.org/licenses/by-nd/2.0/ or send a
letter to Creative Commons, 171 Second St, Suite 300, San Francisco, CA 94105, USA, or Eisenacher Strasse 2, 10777 Berlin, Germany 